\title{
Slug-bubble regime identification in a square channel using a IR Sensor
}

\author{
Aafrin Sulthana. $\mathrm{S}^{1}$, Marieswaran. $\mathrm{T}^{1}$, Braghadesh. $\mathrm{N}^{1}$, Mithran. $\mathrm{N}^{1}$, Venkatesan. $\mathrm{M}^{1}$ * \\ ${ }^{1}$ School of Mechanical Engineering, SASTRA Deemed University, Thanjavur 613401, Tamil Nadu, India
}

\begin{abstract}
Design of micro thrusters for nano satellites, require a detailed understanding of multiphase flow phenomena in micro/mini-channels. This work focuses on the experimental and numerical investigation of an Infra-red sensor behavior during two phase flow of a slug-bubble train (air-water twophase flow). The regime flows inside a square channel of sides $2 \mathrm{~mm}$ and $0.5 \mathrm{~mm}$ thickness made of borosilicate glass. The interference of the slug-bubble train flow pattern on the IR transceiver characteristics is experimentally studied as current signals corresponding to the number of photons received by the photodiode. A numerical model is developed to analyze the IR transceiver characteristics using COMSOL Multiphysics package. The experimental and numerical results are in good agreement and the developed system with proper calibration can be used to design feedback loops for micro thrusters.
\end{abstract}

\section{Introduction}

Capillary gas-liquid two-phase flow is prevalent in many applications for industries including nuclear reactors and modern micro-chemical propulsion systems. The thickness of liquid film surrounding bubble and slug regime play a significant role in deciding two-phase flow characteristics. The film thickness randomly varies for other flow regimes like stratified, churn and annular flows. Many of the liquid propellants, for space applications are mixed with an oxidizing agent before undergoing combustion. Precise knowledge of liquid film-thickness is required to have an efficient design of micro-thrusters capable of producing precise thrust of the order of few Newtons. Previous works reported on twophase flow characterization is discussed in this section.

Particle image velocimetry (PIV) was employed by Thulasidas et al. [1] to study the bubble-train flow regime inside a capillary tube of circular and square cross-section. The velocity profile, circulating vortices and circulation time were analysed theoretically and experimentally. Mehta and Khandekar [2] measured heat transfer coefficient values during Taylor bubble/slug train flow in mini-channel using IR thermography. The results detailed that the heat transfer was enhanced by 2.5 times during a bubble flow when compared with that of a fully developed laminar single-phase flow. Barreto et al. [3] analyzed flow of air-water in a vertical microchannel of $1.2 \mathrm{~mm}$ diameter. Shi and Wang [4] attempted to measure the water velocity in air-water flow as a function of voltage induced in a inductive flow sensor with electrodes which are arc-shaped.

Greenfield et al. [5] numerically studied multiphase flow inside supersonic micro-nozzles and analyzed the droplet impact on the thrust produced. Liu and Pan [6] studied heat transfer during flow boiling inside a microchannel using infrared thermography. The measured temperatures were observed to be within the theoretical predictions. Air-water flow distribution inside a serpentine mini-channel was analyzed by Malhotra et al. [7] and the pressure drop was studied for different flow regimes. Identification of two-phase flow regimes was done with a IR sensor by Arunkumar et al. [8]. A numerical model was developed using VOF method and validated with experiments. The effect of orientation of IR Transceiver during gas-liquid twophase flow was analysed by Mithran and Venkatesan [9]. The results showed that keeping the transceiver at $90^{\circ}$ orientation gave better results.

The works mentioned above details various studies reported on two-phase flow identification and sensors developed. Also, very few works on two-phase flow behavior characterization using IR sensor were reported in the past. In literature, when compared to other methods which are used to predict the flow regimes, IR sensor could provide more accuracy and is a non-intrusive type. In the current work, along with an experiment, a numerical model is developed using COMSOL Multiphysics package to study the irradiation behavior of infrared rays while passing through a slug-

Corresponding author: mvenkat@mech.sastra.edu, 
bubble two phase flow regime. The experimental and numerical results are compared and the results match reasonably well.

\section{Experimental Setup}

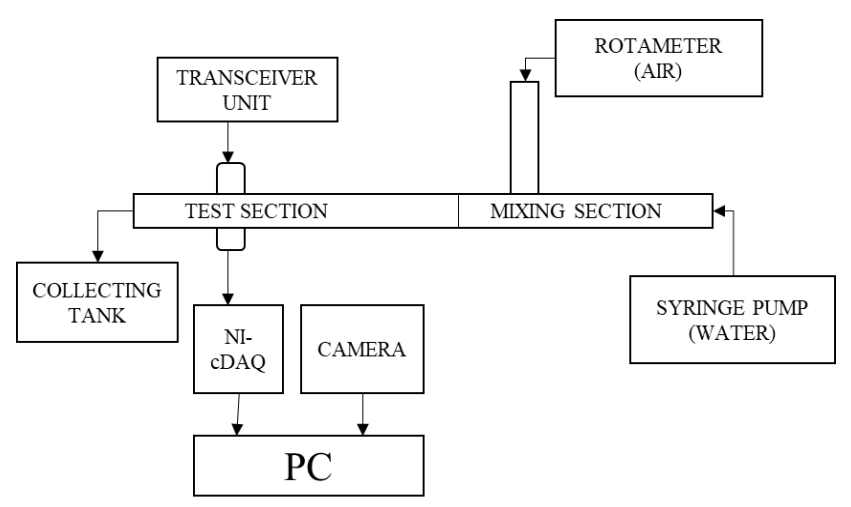

Fig. 1. Experimental setup

The experimental setup shown in Fig. 1 consists of a square channel of $2 \mathrm{~mm}$ side and $0.5 \mathrm{~mm}$ thickness made of borosilicate glass of length $15 \mathrm{~cm}$. A T-section is used for mixing of two separate fluids. The regimes are formed for combination of various flow rates which are measured using rotameter. Air is pumped using a 240 LPH, $3 \mathrm{~W}$ air pump. A syringe pump (E-spin SPK101 model) is used to supply water of TDS concentration of $400 \mathrm{ppm}$ at room temperature. The IR transceiver unit used in this study consists of IR 333-A transmitter and IR PD333-3B photodiode. 1.5 V DC power from AA battery is supplied to the IR LED which emits IR rays. The test section is placed between the IR transmitter and receiver. The IR photon rays pass through the test section and flow regimes having different refractive index which tends to refract some of the photons. The IR transceiver unit is kept vertically perpendicular to the channel, almost touching the outer surface of the tube. The received photon particles are converted into electrical signals by the photodiode and these signals are fed into a NI-cDAQ 9174 chassis with NI 9203 module. The module records the current signals in a computer. The two-phase flow in test section is recorded with the help of a AOS PROMON 501 high-speed camera with 7000 NAVITAR zoom lens. The flow is analyzed frame by frame with AOS studio and the slug-bubble train regime in the two-phase flow is captured. The size of the flow regime is measured with the help of imtool in image processing tool box of MATLAB. The obtained dimensions are used to develop a similar slug-bubble train in COMSOL Multiphysics

\section{Numerical modelling}

A numerical model is developed as shown in Fig. 2 using COMSOL Multiphysics to analyze the incident radiation behavior of the IR sensor which is same as that of the experimental setup.

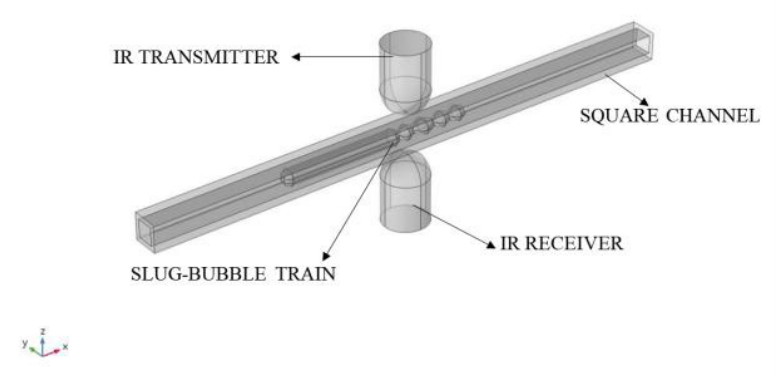

Fig. 2. Representation of numerical model

The refraction of the IR rays follow the Snell's law which postulates that the ratio of the incidence angle to refraction of photon ray is proportional to the ratio of the refractive indices of the two mediums through which the photon rays pass through.

$$
\sin \theta_{1} / \sin \theta_{2}=n_{2} / n_{1}
$$

Where, $\theta_{l}$ - angle of incidence;

$\theta_{2}$ - angle of refraction;

$n_{1}$ - refractive index of the second medium;

$n_{2}$ - refractive index of the second medium.

The number of rays emitted from the source point is set as 100 (which can be varied) to predict the percentage of rays received by the receiver directly. A plane corresponding to the photo-diode cross-section is chosen as a freezing plane to collect the photons that enter the receiver after refracting through a different medium. The thickness of liquid film at the top will be thin when compared to the liquid film thickness at bottom due to the effect of gravity. However, in the developed model, an average uniform liquid film thickness around the voids is considered so that the interpretation of the resulting signal is easier. The model is designed such that the water medium remains stationary while the slug-bubble train sweeps an appropriate distance equivalent to the experimental flow rate.

\section{Results and discussion}

The high speed photograph of slug-bubble train obtained during the experiment is shown in Fig. 3 (a). The corresponding IR transceiver output in terms of current (mA) and numerical results in percentage of photons received is plotted. The position of the slug-bubble train regime is shown in X-axis of Fig. 3 (b). 


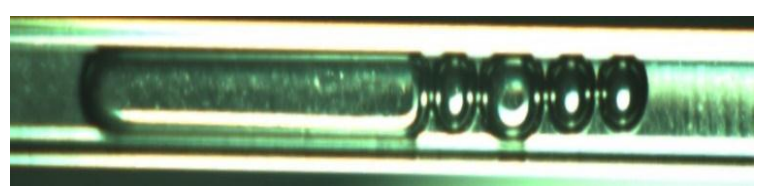

(a)

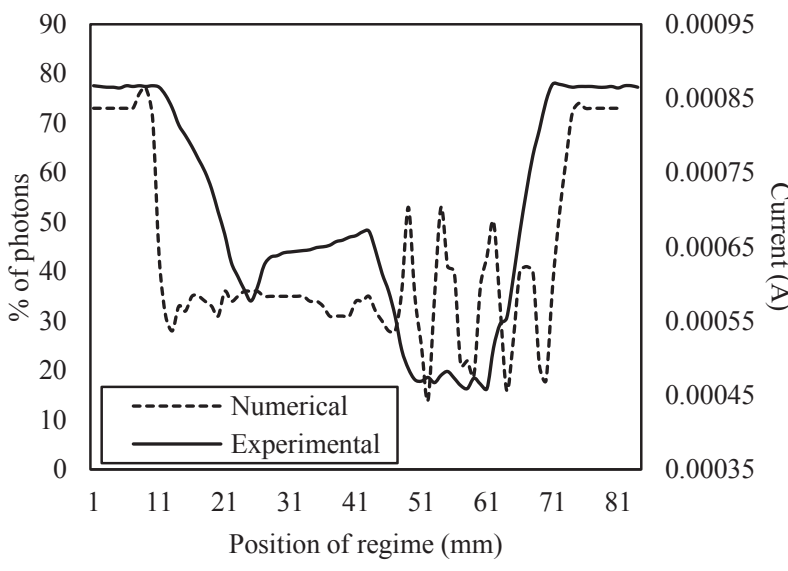

(b)

Fig. 3. Comparison of the experimental and numerical result

The IR rays pass through various mediums like borosilicate glass, water (liquid film) and air (voids), with refractive indices of $1.5,1.33$, and 1 respectively. The scattering of IR rays for different portions of the flow namely, single-phase water, void with liquid film and at the slug-bubble interface are shown in fig. 4(a), 4(b), 4(c).

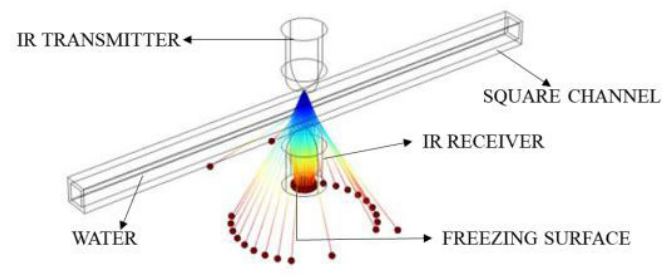

(a)

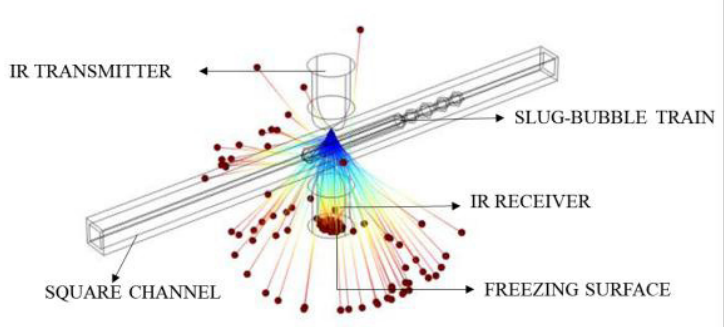

(b)

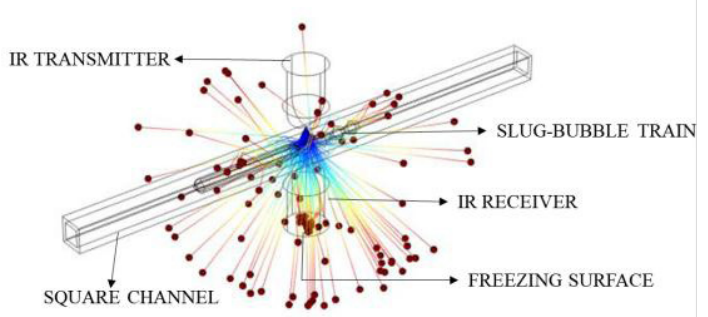

(c)

Fig. 4. 3D scattering of IR rays for test section having (a) only water, (b) slug regime and (c) at the point of slug-slug interference.

The number of photons received for the three cases are 73,31 , and 14 and the corresponding current values are $0.864,0.684$ and $0.471 \mathrm{~mA}$ respectively. For a single slug, the pattern obtained for IR sensor is explained by Mithran [9]. The length and shape of the voids also determine the variations in the number of photons received at the photo-diode. As the length of the voids decreases, like in bubbly flows, the obtained signal pattern in the plot seems to have narrow troughs. The variations in the current measured from IR sensor for the regime considered in the present study is due to the interference during refraction of the rays by the successive slugs. At the point of contact of the two successive slugs, the scattering of the rays is more when compared to other cross section of void fractions. The numerical results are in good agreement with that of the experiments. The velocity at which this two-phase flow crossed the transceiver unit may also be a reason for minor ambiguity between experimental and numerical plots. The results obtained confirm that the IR transceiver setup can be successfully employed for the characterization of slug train flow regimes. Further the numerical model developed can also be successfully employed to analyse various other flow parameters like variation in liquid film thickness, flow shape effects on IR transciever which is the scope for future work. On further extending the study, a feedback method could be developed for precise control of micro thrusters online.

\section{Conclusion}

In present work, the behavior of a IR sensor during airwater two-phase flow for a slug-bubble train flow regime in a square mini channel is analysed. The IR rays effects on air-water two-phase flow measured as current (mA) (experiment) is compared with that of percentage of photons (numerical). The experimental results are compared with the numerical results and validated successfully. The flow inside a mini/microchannel holds a significant role in outer space propulsion systems and many other miniaturized reactors. With precise calibration, these sensors could be used for the flow regime identifications in MEMS. 


\section{References}

1. T. C. Thulasidas, M. A. Abraham, and R. L. Cerro, Chemical Engineering Science, vol. 52(17), 29472962, (1997).

2. B. Mehta and S. Khandekar, 11th International Conference on Quantitative InfraRed Thermography, 11-14 June (2012), Naples Italy.

3. E. X. Barreto, J. L. G. Oliveira, and J. C. Passos, Experimental Thermal and Fluid Science, vol. 63, 1-8, (2015).

4. Y. Y. Shi and M. Wang, Flow Measurement and Instrumentation, vol. 41, 90-96, (2015).
5. Benjamin Greenfield, William Louisos, and Darren Hitt, 49th AIAA Aerospace Sciences Meeting including the New Horizons Forum and Aerospace Exposition, Aerospace Sciences Meetings, https://doi.org/10.2514/6.2011-189

6. T. L. Liu and C. Pan, Applied Thermal Engineering, vol. 94, 568-578, (2016).

7. S. Malhotra, A. Deshpande, and S. Ghosh, International Journal of Multiphase Flow, vol. 96, 48-55, (2017).

8. S. Arunkumar, J. Adhavan, M. Venkatesan, S. K. Das, and A. R. Balakrishnan, Flow Measurement and Instrumentation, vol. 51, 49-54, (2016).

9. N. Mithran and M. Venkatesan, Flow Measurement and Instrumentation, vol. 58, 12-20, (2017). 